\title{
An Initial Memory Model for Virtual and Robot Companions Supporting Migration and Long-term Interaction
}

\author{
Wan Ching Ho, Mei Yii Lim, Patricia A. Vargas, Sibylle Enz, Kerstin Dautenhahn and Ruth Aylett
}

\begin{abstract}
This work proposes an initial memory model for a long-term artificial companion, which migrates among virtual and robot platforms based on the context of interactions with the human user. This memory model enables the companion to remember events that are relevant or significant to itself or to the user. For other events which are either ethically sensitive or with a lower long-term value, the memory model supports forgetting through the processes of generalisation and memory restructuring. The proposed memory model draws inspiration from the human short-term and long-term memories. The short-term memory will support companions in focusing on the stimuli that are relevant to their current active goals within the environment. The long-term memory will contain episodic events that are chronologically sequenced and derived from the companion's interaction history both with the environment and the user. There are two key questions that we try to address in this work: 1) What information should the companion remember in order to generate appropriate behaviours and thus smooth the interaction with the user? And, 2) What are the relevant aspects to take into consideration during the design of memory for a companion that can have different types of virtual and physical bodies? Finally, we show an implementation plan of the memory model, focusing on issues of information grounding, activation and sensing based on specific hardware platforms.
\end{abstract}

\section{INTRODUCTION}

Memory is essential to any social being. The same argument applies to artificial companions ${ }^{1}$ that could establish long-term relationships with human users. One usual problem in the interaction with artificial companions is that users tend to lose interest rapidly due to lack of 'life' and unmet expectations of the companion's intelligence and responsiveness. User motivation for interaction decreases with time as companions continue to perform pre-defined rigid sets of repetitive behaviours, leading to user frustration.

This problem must be tackled in order to prolong and produce a more engaging and natural interaction between the artificial companion and the user. From the perspective of social intelligent companions or agents, Dautenhahn [1] argues that the better computational agents can meet our human cognitive and social needs, the more familiar and natural they are, and the more effectively they can be used as

Wan Ching Ho and Kerstin Dautenhahn are with Adaptive Systems Research Group, School of Computer Science, University of Hertfordshire, UK. E-Mail: \{w.c.ho,k.dautenhahn @herts.ac.uk. Mei Yii Lim, Patricia A. Vargas and Ruth Aylett are with Department of Computing Science, Heriot-Watt University, Edinburgh, UK. E-Mail: $\{$ p.a.vargas, myl, ruth $\} @$ hw.ac.uk. Sibylle Enz is with Otto-Friedrich Universitaet Bamberg, Germany. E-Mail: sibylle.enz@uni-bamberg.de

${ }^{1}$ In this paper the general use of the term 'artificial companion' refers to both virtual companions and robot companions. tools. Hence, we believe that memory is vital if the artificial companion is to be capable of learning and adapting itself to the environment.

Modelling a human-like memory has always fascinated AI researchers and led to various memory models contributing to the understanding of human cognition [2]. In the earlier years, modelling the major characteristics of these memories allowed intelligent programs to remember situations as cases and, most importantly, extracting reasoning rules from these cases stored in a database to extract (see [3] for an overview). 'Scripts' from Schank and Abelson [4], also captures two important aspects of human memory in the perspective of developmental psychology for it represents everyday events and activities, and it has social and cultural components. In particular, recent research emphasising the role of episodic memory on a cognitive robot [5] and on simulated agent [6], [7] architectures have achieved fruitful results through the learning of temporally sequenced episodes/events, or remembering the 'significance' of events (through creating impact to agent's internal states).

In addition to modelling the cognitive aspect of human memory, the embodiment of an artificial companion must also be taken into account while developing the memory architecture. Nowadays artificial companions can be embodied in real social environments with different types of innovative technologies (e.g. physical robots, social toys or graphical synthetic characters) with which users can interact daily. This had led to research in which an artificial companion can migrate seamlessly among these potentially useful platforms in order to maintain the best companionship with the user, reducing the user's cognitive load in adapting him/herself towards these technologies [8], [9].

Following these research directions, here we propose an initial memory model for a long-term artificial companion that can potentially 1) migrate between virtual and robotic embodiments and 2) preserve a long-term relationship with individual users in order to provide coherent interaction without loss of consistency or continuity.

There are two key questions that we try to address in this work. First, what information should an artificial companion remember in order to generate appropriate behaviours and thus smooth the interaction with the human user? To the best of our knowledge, a second question has not yet been proposed in the area of human-robot interaction. It concerns the potential migrating nature of an artificial companion and how to design a generic memory model for a companion which can have different types of virtual and physical bodies.

The rest of this paper is organised as follows: first 
we will discuss relevant background research into human memory, specifically the nature of remembering and forgetting processes. Next we will review literatures on existing computational models of human short-term and long-term memory, in particular autobiographic memory. Following that we will illustrate the proposed initial memory model for artificial companions that we are currently developing in the LIREC $^{2}$ project. Finally we will discuss issues relating to the migration process allowing companions to be embodied in various virtual and robotic platforms, and the grounding of experiences for robot companions.

\section{Models of Human Memory}

The human memory works on the basis of three different processes. First, information from the external and internal sensory system of the organism is encoded. Second, the information is stored either in the short-term memory (STM) or long-term memory (LTM), as will be further described later in this section. Finally, the information can be remembered or retrieved (if it has not been forgotten). Note that it is as yet unclear and controversial among scientists, how exactly human memory works [10], [11], [12].

\section{A. Short Term Memory (STM)}

Providing an interface between perception, LTM and action, STM is a limited capacity system, which temporarily maintains and stores information perceived from the surrounding environment. In recent years STM has been considered an important component to model to enhance the ability of a software companion or physical robot to learn new skills and tasks through utilising past short-term experiences. There are two complementary approaches that make use of the characteristics of STM:

- Control of attention in memory: focusing on the most relevant features of the current task and allowing for more robust behaviour in the presence of distracting or irrelevant events. This was shown to be an effective way of limiting the search space for perceptual systems [13].

- Utilising STM contents in similar ways to those originally developed for LTM: supporting learning that generalises across different tasks. STM can retain a limited amount of information in a temporal sequence for a rehearsal process to determine whether an item is to be remembered [14], [15]. This can avoid the 'out of sight, out of mind' problem caused by immediate occlusions or confusions.

\section{B. Long-term Memory (LTM)}

LTM keeps a large quantity of information for potentially a very long time. Information stored in LTM can be of very different types. According to one of the existing models of the human memory [16], LTM can be divided into declarative and procedural memory. The declarative memory is formed of a semantic and an episodic memory. Autobiographic memory (AM), which is the focus of our research, is a

\footnotetext{
${ }^{2}$ The LIREC Project (LIving with Robots and InteractivE Companions), http: //www. lirec.org/
}

specific kind of episodic memory that contains significant and meaningful personal experiences for a human being [17], [18]. The organisation of a human AM has a hierarchical structure, in the sense that memories of single events are nested within larger cognitive structures [19], [20]. Life events [18] together with events associated with emotions, indicate that central knowledge structures relating to the self have been employed in representing AM. It is believed that AM serves a central function in providing the basis for social interaction, and maintenance of a dynamic self-concept as well as the representation of the meaning of concepts [21].

Moreover, two features of autobiographic memory are generally defined and accepted by researchers in psychology, as pointed out by Conway et al [22]:

- AMs are mental constructions of the self.

- They very often feature imagery while simultaneously containing abstract personal knowledge [18], [23], [24].

\section{Remembering and Forgetting}

As already stated, the three main activities related to memory modelling are: encoding, remembering and forgetting [25]. Information from STM is encoded in LTM through repeated exposure and generalisation. Remembering or retrieval involves recall and recognition while forgetting may be caused by several processes [26].

Forgetting, in particular, is essential and useful for if we were to record every bit of incoming information, we would have information overload, difficulty in organizing it and in focusing on one piece of information at a time. Hence, a number of theories of forgetting have been developed by neuroscientists and psychologists, which aim at explaining these mechanisms, and thus why we forget. The most relevant for our investigation purposes are trace or functional decay theory and repression.

Functional decay theory [27] has been found to be useful in making quantitative predictions for human performance in dynamic task environments. It suggests that encoding and decay are critical to maintaining situational awareness in an environment where tasks change continually. The cognitive system must be prepared to forget so that resources can be concentrated on the current state of the world. The core idea of this theory is that the most recent information must be the most active in memory to allow reliable and fast retrieval. As the current task decays, retrieval becomes more difficult. However, decay through use is minimal, in other words, the more active an item, the more accurately and quickly it can be retrieved from memory. The amount of time invested during encoding determines the amount of decay during use.

Repression occurs when memories are unconsciously blocked from our awareness. It could be seen as the purposeful but subconscious block of memories. These strategies to 'forget' disturbing experiences have been researched in psychoanalysis as defence mechanisms, strategies that serve to protect the self from situations and emotions with which one cannot cope [28]. In case of these unconscious or conscious strategies of motivated forgetting, remembering, discussing or rehearsing memories are important techniques 
to strengthen the retrieval of the suppressed or repressed memories. Similarly, forgetting details of disturbing events might also be due to the fact that disturbing events are simply less often discussed and rehearsed than positive memories.

In our proposed memory model, it is envisage that an artificial companion will have the capability to remember and forget information perceived from its interaction environment so that it can update and adapt its memory accordingly. By constantly reconstructing memory, e.g. using remembering and forgetting mechanisms, the companion will be able to learn to behave in an appropriate way because its attention can be focused on important information relevant to the current interaction situation.

\section{ROBOTS AND VIRTUAL CHARACTERS AS COMPANIONS}

So far, the idea of a robot or a virtual character companion has not been widely accepted and sometimes not even considered or imagined. Several matters should be addressed in order to facilitate long-term human interaction with virtual or robot companions. Among these we can highlight ethical issues, grounding problems and potential migration concerns.

\section{A. Ethical Issues}

Long-term interaction with virtual or robot companions, which involves data storage of personal information, naturally raises ethical issues as a primary concern. In a previous work Vargas and collaborators [26] focused on what the artificial companion should and should not forget and its consequences when taking into consideration ethical concerns. The authors suggested the creation of a master Roboethical [29] theory, which would encompass all positive features of each type of ethical theory. In our model we attempt to follow these guidelines towards the conception of a Roboethical memory.

\section{B. Grounding Problem}

The problem of grounding has been a difficult problem existing in the research field of embodied AI for decades. In the LIREC project, our main concern is what defines an experience for an embodied companion.

Any embodied companion, in particular a robot companion, situated and acting in an environment will have many sensors through which it can receive information about itself and its environment. Some of them sense the external environment (e.g. visual, infra-red, sonar sensors), while others sense the internal environment and body (e.g. motor position, internal temperature sensors, gyroscopic accelerometers) and yet others still sense internal variables (e.g. variables that simulate motivational or affective states). A number of these quantities are naturally discrete, like buttons and switches. However, in general the observed quantity is continuous and in current robotic systems the sensor maps the continuous values into discrete observations to some level of precision [30].

\section{Migration}

Some researchers have explored artificial companions that can migrate their 'minds' through different bodies. Ono et al [31] proposed the term companion migration and they experimentally verified the process for smooth communications between humans and robots. In a later work, Ono and his colleagues extended the design to create the ITACO system which aims at achieving companion's 'ubiquitous cognition' and does support a wider variety of human robot interaction (HRI) contexts (e.g. in [32], [8]).

Similar to the ITACO system but with the mixture of virtual and robotic companions, companion Chameleons [33] simulates the environment and behaviours of a chameleon and illustrates companions that migrate and mutate within and between robotic and virtual platforms.

One of the important aspects of agent migration is the ability of the agent to maintain its 'identity'. To investigate this, Koay et al [9] carried out an experiment on the visual realisation of migration process - whether the users believe that they are still interacting with the 'same agent' even after it migrates from a humanoid robot to a zoomorphic robot platform.

The memory design for companions migration remains unexplored as the previous studies have not included the notion of long-term interaction - therefore LTM was not considered as part of their research and related experiments.

\section{RELATED WORK}

Computational models of AM have not so far adequately accounted for its hierarchical organisation and nested structure or fully exploited these characteristics. According to Ho et al. (2008), so far a comprehensive model of autobiographic memory has not been implemented and tested thoroughly in believable and possibly conversational graphical companions, not to mention real robot companions.

In recent years, the use of temporal sequences of episodic events has been rapidly growing in both robot and virtual companions' research areas. For example, by collecting relevant events stored in episodic memory, an exploration robot is able to reduce its state-estimate computation in localising itself and building a cognitive map in a partially observable office environment [34]. Also, long-term episodic memory with attributing emotions may help a virtual robot to predict rewards from human users, thus facilitating human-robot interactions in a simple Peekaboo communication task [35].

Mirza et al [36] uses the concept of interaction histories, defined as the "temporally extended, dynamically constructed and reconstructed, individual sensori-motor history of an companion situated and acting in its environment including the social environment". This work is strongly inspired by dynamical systems approaches to memory and sensori-motor coordination.

The current research trend towards modelling a complete human episodic memory, e.g. episodic memory in Soar [6] and a generic episodic memory module [37], establishes a common structure that consists of context, contents and 
outcomes/evaluation for companions to remember past experiences. These models were created to focus on the following three different aspects:

1. Accuracy - how relevant situations can be retrieved from the memory

2. Scalability - how to accommodate a large number of episodes while not decreasing significantly the performance of the system

3. Efficiency - how to optimise the storage and recall of memory contents

Brom et al [38] attempted to create a full episodic memory storing more or less everything happening around the companion for the purpose of storytelling. The authors claimed that the modelled episodic memory can answer specific questions from human users in real time regarding the companion's personal histories. With the story scenario which was used in their paper, this memory allowed an companion to describe past actions in time. Forgetting processes were also partially implemented in their work - in the companion's LTM records, less emotionally interesting records were deleted.

Furthermore, previous research in [7] aimed at modelling the psychological concept of AM computationally and integrated it into a synthetic companion architecture. With this memory included, companions are not only capable of recognising and ranking significant events which originate in the companions' own experiences, but can also remember, recall and learn from these experiences. Thus companions' believability can be increased and the interactivity of the software can be more fulfilling for the user [7].

Different types of computational memory architectures for Artificial Life autobiographic companions have also been developed and experimentally evaluated in other works. For an overview, see [39], [40]. These architectures include typical human memory modules which are commonly acknowledged in psychology: short-term, long-term and positively and negatively categorised memories.

Forgetting has also been adopted in many learning algorithms. Ishikawa applied structural learning with forgetting [41] to two of three phases: 1) learning with forgetting, 2) hidden units clarification and 3) learning with selective forgetting. In the first phase, connection weights are constantly decayed so that unnecessary connections can be eliminated and a skeletal network emerges. However, this step may result in a mean square error that is larger than that by back propagation learning. Therefore, in the learning with selective forgetting phase, only the connection weights whose absolute values are below a certain threshold are decayed. The summation is restricted only to weak connections making the mean square error much smaller than that in learning with forgetting. The determination of the amount of forgetting is important to ensure efficient learning because if it is too large, even necessary connections fade away while if it is too small, unnecessary connections remain, resulting in a network far from skeletal.

Koychev [42] utilises a gradual forgetting method in learning drifting concepts by applying a time-based forgetting function. The idea is comparable to functional decay theory that suggests the most recent information is the most active in memory. Some changes are required to existing induction learning algorithms (eg. NBC, ID3) that treat all training examples as equally important to include a weight for the examples according to its occurring time. By doing so, the last observations become more significant for learning algorithms than the old ones. The result of experiments showed an improved predictive accuracy and adaptability of the systems that adopt learning algorithms with gradual forgetting.

\section{Initial MEMORy MOdel}

In this section we propose our design of an initial memory model for migrating artificial companions. Taking into account different aspects from the existing computational memory models, here we aim at addressing the adaptability of an artificial companion to preferences of the user as well as the dynamic environment, thus facilitating the long-term interaction.

By modelling certain features, which reflect on the general characteristics of human autobiographic memory and general event representation (GER), our memory model covers different aspects of information processing from low-level to high-level for artificial companions.

The remainder of this section introduces the features of each component separately and discusses in detail the lowlevel design which supports the migrating process for this memory model.

\section{A. High-Level Design}

Computationally, memory can be modelled as a succession of three different stores, one for the sensory information, the second for STM, and the last for LTM; the above described processes, encoding, storing, and retrieving, work on these three entities.

Figure 1 shows an overview of the complete memory model. The model consists of components of LTM, STM, Reasoning and Action Selection, Actuation and Sensing.

To facilitate the long-term interaction between the artificial companion and the user in scenarios within the LIREC project, the memory model needs to capture the user's everyday routine activities as well as the knowledge about the environment for the processes of goal formulation and accomplishment. Moreover, the enhanced LTM and STM in our model are able to cope with 'organic' developmental processes, such as learning new behaviour and attributing emotions to its LTMs for significant events.

Based on the memory model illustrated in Figure 1, an individual companion can create different behavioural or conceptual meanings for an action or object in its LTM, depending on the way it interacts with the user and the environment.

1) Short-Term Memory: STM can be modelled computationally to maintain an companion's current focus on its interaction world and activated goals that guide behaviour generated by the Reasoning and Action Selection component. 


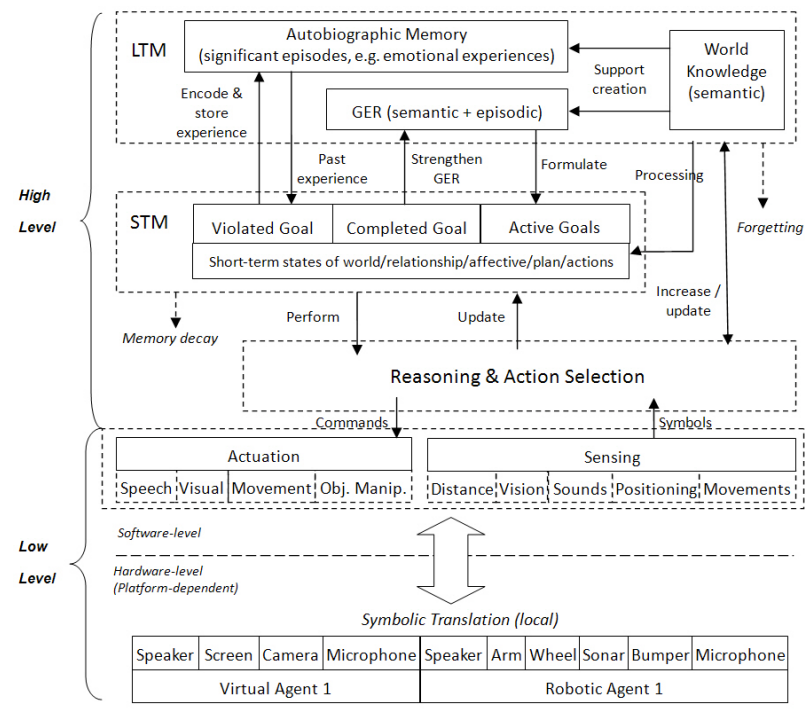

Fig. 1. A generic memory model for migrating artificial companions, with arrows showing the transition of information among different components. Note that italic labels indicate the effect of forgetting.

In Figure 1 we illustrate the goal management in STM, and how different types of information can be retrieved from other components as well as the influences STM can bring to them. Moreover, STM holds all active information relevant to the companion's current and recent goal processing to ensure effective and appropriate reactions to its immediate circumstances.

With the sensory data derived from the fusions of companion's sensors, STM maintains three different types of goals, namely active, completed and violated goals. In order to ensure the adaptation of the companion to the environment, these goals are processed, updated and verified with knowledge supplied continuously from the LTM. Therefore, the goal structure directs the companion's behaviour in a way that complements the companion's previous understanding of the environment.

- Active goals: Active goals are formulated in real-time by General Event Representations (GERs) and supported by World Knowledge (WK) modules from LTM (see next sub-section for details of GERs and WK). They are goals that a companion needs to achieve in a given situation so as to complete a task or satisfy its own motivational states.

- Completed goals: Every active goal is continuously monitored in STM and updated with sensory input. Once the conditions are met and an active goal is achieved, this goal then becomes a completed goal. At the same time this successful occurrence strengthens the same type of goals in GERs - verifying a successful goal will lead to the encoding and further consolidation of the goal in GERs. Therefore, it results in the facts that 1) this goal will be more likely to be chosen for activation in the future, and 2) details of the completed goal will be forgotten by the companion.

- Violated goals: In some cases there will be stimuli that are unexpected or novel to the companion. These stimuli may violate all active goals that the companion tries to achieve currently. The violation of goals leads to the demand for specific past experiences from Autobiographic Memory (AM) in LTM. Since an companion's AMs are constructed from relatively distinctive and emotional experiences in the past (see next sub-section for details), reconstructing these experiences results in updating all active goals in STM, impacting the companion's current emotional states and thus forcing a new regulation of the companion's current behaviour.

These three types of goals maintained by STM are designed to form an iterative loop whose purpose is to reduce the discrepancy between desired and actual goal states. As aforementioned, in performing this discrepancy reduction the behaviour is regulated. Newly activated goals that emerge from AM can further guide selective attention and actions this process is necessary to sustain the goal structure.

When a goal is activated, the companion will construct an appropriate plan to achieve that goal. It keeps track of the progress of the plan and the state of the environment to ensure that its plan is still valid. Sensory data brought in by the component 'Sensing' contains the update of the companion's current environment. Outcomes of current actions success or failure - are noted and alternative actions or plans are established whenever necessary. The companion's current affective states (emotions, mood and/or drives depending on the companion's emotional model) and its relationship with other companions affect its goal activation.

2) Long-Term Autobiographical Memory: To develop artificial companions that interact with human users over a long period of time, LTM is the most important component in our model, ensuring that companions learn and adapt socially over the long-term. Supported by the WK module ${ }^{3}$, the features provided by AM and GERs are our main foci in developing a comprehensive LTM in the LIREC project ${ }^{4}$.

Basically, General Event Representations (GERs) are goal categories organised dynamically and based on the current goal activity [43]. Their goal-oriented categories can be perceived as memory schemata and scripts since they generally encapsulate all of an companion's knowledge of a particular type of object in the world, or a sequence of perceptions and actions of an event. Knowledge for digesting routine goals (i.e. goals that are familiar and do not create emotional

\footnotetext{
${ }^{3}$ Artificial companions will need to be pre-installed (or personalised by the user before starting the mutual interaction) a certain amount of semantic world knowledge in the early stage of a given HRI scenario. This set of semantic world knowledge is necessary to assist basic tasks or interactions that a companion is going to perform, as well as to avoid a user teaching its companion every basic fact of the world at the beginning of the interaction. For instance, the knowledge includes date and time, seasons, user's life routine in different days of a week, objects and resources available in the local environment.

${ }^{4}$ Note that our memory model does not aim at simulating all characteristics of human long-term and autobiographic memory, but it captures essential features from a number of well developed psychological models. This model establishes the inter-relationship between AM, which provide personal knowledge to support STM based on the given goal activity, and GERs for handling routine and highly anticipated events for companions.
} 
impact to the companion) is similar to human semantic knowledge which is used to understand the world. Therefore these event categories provide expectations about what the companion will experience (e.g. seeing, hearing).

When a routine goal from STM is evaluated against existing goals in GERs, if it does not differ from them, it will be 'absorbed' into the GERs through rehearsing the categories and its content details can be decay (be forgotten) overtime. Therefore routine goals do not have great details in GERs but their schematic structure and meaning are preserved.

As discussed above, STM modulates a companion's behaviour and supports LTM construction with knowledge supplied by different modules in LTM. Since STM holds a subset of LTM closely connected with the current goal structure and it always monitors the goal accomplishment, external stimuli perceived by an companion will be examined through a 'filtering' process in the STM component. A new goal category is created when a novel stimulus cannot be absorbed into existing GERs in LTM - the stimulus is 'incomprehensible' by the existing goals and sub-goals in GERs.

Furthermore, goal verification in STM is a critical and dynamic process in maintaining the coherent behaviours of companions, and it requires retrieving a considerable amount of knowledge from AM based on the current ongoing goal activity. Novel and unexpected stimuli, which can be significant to the companion and may create a considerable amount of discrepancy between the standard and current state of the world, will affect the companion's emotional states and take part in forming particular sequence of event in AM.

For instance, in [44], significant events were indicated by a substantial amount of change in companions' internal physiological variables. Moreover, WK also provides semantic knowledge to support the constructions of important events in AM. These features are illustrated in Figure 1.

Events retained in the AM differ in duration and complexity and they can be seen as highly specific and unique experiences. Unlike routine goals in GERs, they also act as organising representations for memories of more specific occurrences. Therefore knowledge constructing these specific events constitutes a central feature in AM.

In summary, both AM and GERs for companion are instrumental in the generation and maintenance of a goal structure. They are also intended to reduce the quantity of information that actually has to be stored and to facilitate a more coherent 'self' for the companion to have more consistent behaviour. In order to ensure that each significant event in AM is meaningful and coherent, WK provides semantic knowledge to support the formation of a complete event.

3) Forgetting: Forgetting is useful to improve the efficiency, scalability and adaptability of cognitive systems operating in dynamic task environments, such as a robot's interaction environment. The issue of privacy and social desirability may also be addressed through these mechanisms. Previous work on the issue of privacy in relation to robot companions [45], [26] has suggested that while there are clear concerns regarding privacy, potential users of companions recognise the need for retention and retrieval of information of a personal nature in order for the robot to effectively perform as a companion. Participants pointed towards mechanisms for 'forgetting' as vital to address this issue.

Therefore, in order to implement such control, our proposed memory model should include forgetting mechanisms by not only utilizing the trace or functional decay theory [46], [47] for STM and LTM but also considering repression. It is believed that these mechanisms will both address the issue of social desirability as well as those of efficiency, scalability and adaptability.

By utilising a decay theory, the idea is that memory traces that are of the immediate past are denser than the old ones. When information is perceived, it enters the STM. With continuous activation through rehearsal or frequent recall this memory may eventually become LTM. However, if the information falls into disuse, the memory trace will start to decay and eventually fade from memory.

The information that receives frequent attention will go through reconstruction processes before it is consolidated as LTM. This is part of the learning process where memory structures are modified continuously based on incoming information to ensure their currency with respect to the world state. By being able to notice and recall differences in experiences, the robot will be able to learn about its environment more effectively. General structures will help the robot in deciding what to pay attention to, and reminding forces it to make use of prior knowledge to form expectations. Care needs to be taken when generalising information to ensure that particular differences that may be valuable are not lost.

A repression mechanism could be implemented in order to allow the user to 'repress' any memory event that might be considered inadequate for storage. Following these guidelines, a robot's memory can be personally tailored to suit particular user needs while initialising the robot. The same memory architecture, with different levels of forgetting and repression mechanisms to handle sensitive contents, can support various user groups with regards to personal privacy.

\section{B. Low-Level Design}

This sub-section addresses the coherence issue of the companion's memory encoding and retrieving processes associated with different embodiments.

While grounding symbols for robotic companions is a difficult challenge, dealing with companions' migration across different platforms is a further one - the user's social engagement must be maintained when an companion's embodiment changes over time. In particular, when an companion migrates, aspects such as affordances, interaction interfaces and behavioural expressions may be affected.

As shown in the bottom part of the memory model in Figure 1, the low-level symbolic translation process which is platform-independent can be perceived as the first initial step towards the answer of a partitioned memory. Here 


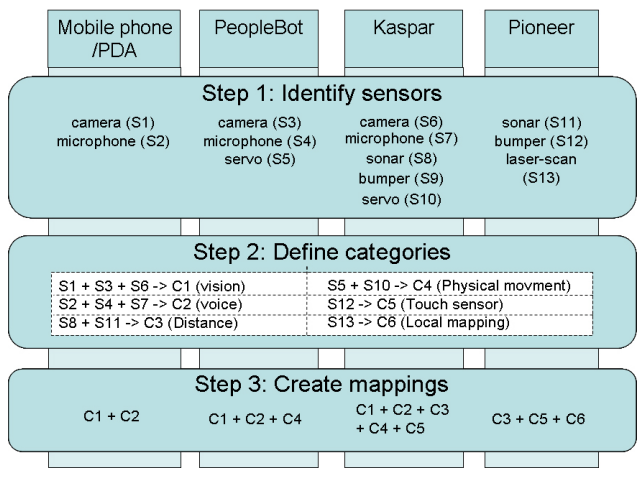

Fig. 2. The low-level symbolic translation process proposed for the initial memory model. Note that PeopleBot ${ }^{\mathrm{TM}}$ and Pioneer ${ }^{\mathrm{TM}}$ robots were developed by Activrobots $\mathbb{R}$. Kaspar is a child-size robot which is capable of demostrating facial expression and expressive postures that was developed by researchers at University of Hertfordshire, see [48] for details.

we pursue the ideas shown in Figure 1, 'Actuation and Sensing', in which a specific embodiment of the artificial companion represents a unique set of interaction histories that the companion possesses.

Take 'Sensing' as an example; three main steps are involved (Figure 2):

1. Identifying the type of sensors (S1, S2, etc) in each hardware platform (companion's embodiment)

2. Then defining sensory categories $(\mathrm{C} 1, \mathrm{C} 2$, etc)

3. Finally a backward mapping is done to physical embodiment of the companion

Through creating 'Sensing' with these three steps, a companion's memory can be partitioned and incrementally encoded based on the specific platform it has migrated to. During the migration process, the companion embodies with the new platform along with the complete memory, and then it retrieves the right set of 'Sensing' from the embodiment to start the encoding process as well as 'Activation' to execute its planning with behaviours that the current embodiment can support.

Multimedia content is thus addressed with 'Activation' and 'Sensing'. However, as symbolic translations are carried out locally in the specific platform and thus memory component above 'Activation' and 'Sensing' can process all types of sensory input and actuator output as symbols, local symbolic translations become a critical process to allow companions to make sense of the surrounding environment as well as to guide its behaviour in changing the environment.

\section{Discussions AND Future WORK}

In this paper we have introduced a comprehensive and generic memory model for artificial companions that adapt to their environment and interact with human users for a long period of time.

In our model, we use a top-down approach for representing the knowledge and its transfer between components. Meanwhile from a bottom-up perspective we investigate the grounding problem of creating meaningful experiences for robots and how companions can maintain a consistent memory system while migrating from one platform to another.
Grounding allows symbols to have meaning for companions (usually robots) - symbols must be grounded in the companion's own interaction with the real world. Targeting this issue, we first discuss how to create LTM in robots, and then we propose a method to implement low-level memory particularly for companions which can migrate to different physical bodies.

Here we suggest starting by considering an companion's memory as a kind of interaction history, as defined in Section II by Mirza [36]. In addition to address the grounding problem, this definition has three key aspects [30]:

- Temporal extension: The overall horizon of an companion's experience extends into the past (including previous experience available to the companion) and also into the future in terms of prediction, anticipation and expectation.

- Dynamic construction: This indicates that the history is continually being both constructed and reconstructed. Previous experiences are modified in both the processes of 'storage' and recall, and potentially affect how new experiences will be assimilated into the history in the future.

- Remembering in action: The process of remembering drives and shapes the choice of current and future actions, while also, itself, dynamically re-shaping the structures employed in remembering.

There is certainly much further low-level specification to be carried out in the near future. Here we have illustrated, in both LTM and STM components, the conceptual design allowing companions to identify, characterise and distinguish experiences for creating a coherent long-term interaction history.

Memory models for virtual companions can be far more sophisticated than those for robots since they have easy access to knowledge about the companion's environment, the companion itself and other companions in the environment (regarding their behaviour, internal states, goals etc.). Such knowledge is not readily available to autonomous robots which rely on their local perception and learning capabilities. Hence, one of the key challenges for us will be to further improve our memory model to accommodate both virtual and robotic companions.

Finally we expect that LIREC artificial companions embedded with this memory model will be able to draw on past experiences to affect their future behaviour - thus will be able to become more believable and affective companions.

\section{ACKNOWLEDGMENTS}

We would like to thank Dr. Kheng Lee Koay at University of Hertfordshire for his comments on agent migration.

This work was partially supported by European Community (EC) and is currently funded by the EU FP7 ICT215554 project LIREC (LIving with Robots and Interactive Companions). The authors are solely responsible for the content of this publication. It does not represent the opinion of the EC, and the EC is not responsible for any use that might be made of data appearing therein. 


\section{REFERENCES}

[1] K. Dautenhahn, "The art of designing socially intelligent agents - science, fiction, and the human in the loop," Applied Artificial Intelligence, vol. 12, no. 7-8, pp. 573-617, 1998.

[2] S. Franklin, "Autonomous agents as embodied ai," Cybernetics and Systems Special Issue on Epistemological Aspects of Embodied AI, vol. 28, no. 6, pp. 499-520, 1997.

[3] J. L. Kolodner, Case-Based Reasoning. San Mateo, CA: Morgan Kaufmann, 1993.

[4] R. C. Schank and R. P. Abelson, Scripts, Plans, Goals and Understanding: An Inquiry into Human Knowledge Structures. Hillsdale, New Jersey: Lawrence Erlbaum Assicuates, 1977.

[5] W. Dodd and R. Gutierrez, "The role of episodic memory and emotion in a cognitive robot," in Proceedings of 2005 IEEE International Workshop on Robots and Human Interactive Communication, 2005, pp. 692-697.

[6] A. Nuxoll and J. Laird, "A cognitive model of episodic memory integrated with a general cognitive architecture," in Internetional Conference on Cognitive Modeling, 2004.

[7] W. C. Ho and K. Dautenhahn, "Towards a narrative mind: Creating coherent life stories for believable agents," in Intelligent Virtual Agent (IVA) 2008, 2008, pp. $59-72$.

[8] K. Ogawa and T. Ono, "Itaco: Effects to interactions by relationships between humans and artifacts," in Proceeding of Intelligent Virtual Agents 2008, 2008, pp. 296-307.

[9] K. L. Koay, D. S. Syrdal, M. L. Walters, and K. Dautenhahn, "A user study on visualization of agent migration between two companion robots," in 13th International Conference on Human-Computer Interaction (HCI 2009), 2009, to appear.

[10] M. Wilson, "Reactivation of hippocampal ensemble memories during sleep," Science, vol. 265, 1994.

[11] C. I. De Zeeuw, "Time and tide in cerebellar memory formation," Current Opinion on Neurobiology, vol. 15, pp. 667-674, 2005.

[12] J. M. Levenson, "Epigenetic mechanisms: a common theme in vertebrate and invertebrate memory formation," Cellular and Molecular Life Sciences, vol. 63, pp. 1009-1016, 2006.

[13] D. Wilkes, M. Tugcu, J. Hunter, and D. Noelle, "Working memory and perception," in IEEE international workshop on robots and human interactive communication 2005, 2005, pp. 686-691.

[14] C. Peters and C. O'Sullivan, "Synthetic vision and memory for autonomous virtual humans," Computer Graphics Forum, vol. 21, no. 4 , pp. $743-753,2002$.

[15] W. C. Ho, K. Dautenhahn, and C. L. Nehaniv, "Computational memory architectures for autobiographic agents interacting in a complex virtual environment: a working model," Connection Science, vol. 20, no. 1 , pp. $21-65,2008$

[16] E. Tulving, "Episodic and semantic memory," in Organization of Memory, E. Tulving and W. Donaldson, Eds. New York: Academic Press, 1972.

[17] K. Nelson, "The psychological and social origins of autobiographical memory," Psychological Science, vol. 4, pp. 7-14, 1993.

[18] M. A. Conway, Autobiographical Memory: An Introduction. Buckingham: Open Univ. Press, 1990.

[19] U. Neisser, "Nested structure in autobiographical memory," in Autobiographical Memory, D. C. Rubin, Ed. Cambridge, UK: Cambridge University Press, 1986, pp. 71-88.

[20] D. B. Wright and J. A. Nunn, "Similarities within event clusters in autobiographical memory," Applied Cognitive Psychology, vol. 14, pp. 479-489, 2000.

[21] K. Dautenhahn, "Embodiment in animals and artifacts," in AAAI FS Embodied Cognition and Action. AAAI Press, 1996, pp. 27-32, technical report FS-96-02.

[22] M. A. Conway, C. W. Pleydell-Pearce, and S. E. Whitecross, "The neuroanatomy of autobiographical memory: A slow cortical potential study (scp) of autobiographical memory retrieval," Memory and Language, vol. 45, pp. 493-524, 2001.

[23] M. A. Conway, "Autobiographical memories and autobiographical knowledge," in Remembering our past: Studies in autobiographical memory, D. C. Rubin, Ed. Cambridge, UK: Cambridge Univ. Press, 1996, pp. 67-93.

[24] M. A. Conway and C. W. Pleydell-Pearce, "The construction of autobiographical memories in the self memory system," Psychological Review, vol. 107, pp. 261-288, 2000.

[25] F. C. Bartlett, Remembering: A Study in Experimental and Social Psychology. Cambridge University Press, 1932.
[26] P. A. Vargas, W. C. Ho, M. Y. Lim, S. Enz, and R. Aylett, "To forget or not to forget: Towards a roboethical memory control," Proceedings of the AISB-2009, p. To appear, 2009.

[27] E. Altmann and W. Gray, "Managing attention by preparing to forget," Human Factors and Ergonomics Society Annual Meeting Proceedings, Cognitive Ergonomics, vol. 1, no. 4, pp. 152-155, 2000.

[28] A. Freud, The Ego and the Mechanisms of Defence. London: Hogarth Press and Institute of Psycho-Analysis, 1937.

[29] G. Veruggio and F. Operto, "Roboethics: a bottom-up interdisciplinary discourse in the field of applied ethics in robotics," International Review of Information Ethics, vol. 6, pp. 3-8, 2006.

[30] N. Mirza, "Grounded sensorimotor interaction histories for ontogenetic development in robots," Ph.D. dissertation, University of Hertfordshire, 2008.

[31] T. Ono, M. Imai, and R. Nakatsu, "Reading a robot's mind: a model of utterance understanding basedon the theory of mind mechanism," Advanced Robotics, vol. 14, no. 4, pp. 311-326, 2000.

[32] T. Ono, "Itaco: Ubiquitous cognition achieved by migratable agents between media," in Proceedings of 10th International Conference on Virtual Systems and Multimedia (VSMM2004), 2004, pp. 1177-1182.

[33] G. O'Hare and B. Duffy, "Agent chameleons: Migration and mutation within and between real and virtual spaces," in the society for study of artificial intelligence and the simulation of behaviour (AISB 02), 2002.

[34] Y. Endo, "Anticipatory robot control for a partially observable environment using episodic memories." Georgia Tech Mobile Robot Lab, Tech. Rep., 2007, gIT-IC-07-03.

[35] M. Ogino, T. Ooide, A. Watanabe, and M. Asada, "Acquiring peekaboo communication: Early communication model based on reward prediction," in Proceedings of IEEE International Conference in Development and Learning (ICDL), 2007.

[36] N. Mirza, C. Nehaniv, D. Dautenhahn, and R. te Boekhorst, "Interaction histories: From experience to action and back again," in Proceedings of the 5th IEEE International Conference on Development and Learning (ICDL), 2006.

[37] D. Tecuci and B. Porter, "A generic memory module for events," in Proceedings to the 20th Florida Artificial Intelligence Research Society Conference (FLAIRS 20), Key West, FL., 2007.

[38] C. Brom, K. Peskova, and J. Lukavskyz, "What does your actor remember? towards characters with a full episodic memory," in International Conference on Virtual Storytelling, ser. Lecture Notes in Computer Science, M. Cavazza and S. Donikian, Eds., no. 4871, 2007, pp. 89-101.

[39] W. C. Ho, "Computational memory architectures for autobiographic and narrative virtual agents," Ph.D. dissertation, University of Hertfordshire, 2005.

[40] W. C. Ho, K. Dautenhahn, and C. L. Nehaniv, "A study of episodic memory-based learning and narrative structure for autobiographic agents," in Proceedings of Adaptation in Artificial and Biological Systems, AISB 2006 conference, vol. 3, 2006, pp. 26-29.

[41] M. Ishikawa, "Structural learning with forgetting," Neural Networks, vol. 9, no. 3, pp. 509-521, 1996.

[42] I. Koychev, "Gradual forgetting for adaptation to concept drift," in In Proceedings of ECAI 2000 Workshop Current Issues in SpatioTemporal Reasoning, 2000, pp. 101-106.

[43] K. Nelson, Event knowledge and cognitive development. London: Lawrence Erlbaum Associates, 1986, pp. 1-19.

[44] W. C. Ho and S. Watson, "Autobiographic knowledge for believable virtual characters," in Proceedings of Intelligent Virtual Agents 2006 (IVA 2006). Springer LNAI, 2006, pp. 383-394.

[45] D. Syrdal, M. Walters, N. R. Otero, K. Koay, and K. Dautenhahn, "He knows when you are sleeping - privacy and the personal robot," in Technical Report from the AAAI-07 Workshop: W06 on Human Implications of Human-Robot Interaction, 2007.

[46] J. Brown, "Some tests of the decay theory of immediate memory," Quarterly Journal of Experimental Psychology, vol. 10, pp. 12-21, 1958.

[47] E. M. Altmann and W. D. Gray, "Managing attention by preparing to forget," Human Factors and Ergonomics Society Annual Meeting Proceedings, vol. 1(4), pp. 152-155, 2000.

[48] B. Robins, K. Dautenhahn, and P. Dickerson, "From isolation to communication: A case study evaluation of robot assisted play for children with autism with a minimally expressive humanoid robot," in Proc. the Second International Conferences on Advances in ComputerHuman Interactions, ACHI 09, 2009. 